\title{
Spatial and Temporal Sentiment Analysis of Twitter data
}

\author{
Zhiwen Song and Jianhong (Cecilia) Xia ${ }^{*}$ \\ Department of Spatial Sciences, Curtin University \\ *c.xia@curtin.edu.au
}

\begin{abstract}
The public have used Twitter world wide for expressing opinions. This study focuses on spatio-temporal variation of georeferenced Tweets' sentiment polarity, with a view to understanding how opinions evolve on Twitter over space and time and across communities of users. More specifically, the question this study tested is whether sentiment polarity on Twitter exhibits specific timelocation patterns. The aim of the study is to investigate the spatial and temporal distribution of georeferenced Twitter sentiment polarity within the area of 1 $\mathrm{km}$ buffer around the Curtin Bentley campus boundary in Perth, Western Australia. Tweets posted in campus were assigned into six spatial zones and four time zones. A sentiment analysis was then conducted for each zone using the sentiment analyser tool in the Starlight Visual Information System software. The Feature Manipulation Engine was employed to convert non-spatial files into spatial and temporal feature class. The spatial and temporal distribution of Twitter sentiment polarity patterns over space and time was mapped using Geographic Information Systems (GIS). Some interesting results were identified. For example, the highest percentage of positive Tweets occurred in the social science area, while science and engineering and dormitory areas had the highest percentage of negative postings. The number of negative Tweets
\end{abstract}

\section{How to cite this book chapter:}

Song, Z and Xia, J. 2016. Spatial and Temporal Sentiment Analysis of Twitter data. In:

Capineri, C, Haklay, M, Huang, H, Antoniou, V, Kettunen, J, Ostermann, F and Purves, R. (eds.) European Handbook of Crowdsourced Geographic Information, Pp. 205-221. London: Ubiquity Press. DOI: http://dx.doi.org/10.5334/bax.p. License: CC-BY 4.0. 
increases in the library and science and engineering areas as the end of the semester approaches, reaching a peak around an exam period, while the percentage of negative Tweets drops at the end of the semester in the entertainment and sport and dormitory area. This study will provide some insights into understanding students and staff's sentiment variation on Twitter, which could be useful for university teaching and learning management.

\section{Keywords}

spatial and temporal analysis; sentiment analysis; Twitter; georeference

\section{Introduction}

Twitter as one of vital platforms for people to publically express their opinions and feelings about events and their private lives, has attracted enormous attention with millions of followers (Li et al. 2013). Numerous studies have been conducted on opinion mining and sentiment analysis on Twitter (Pang \& Lee 2008; Poria et al. 2014; Taboada et al. 2011; Liu 2012). The sentiment classification methods have been developed from simple text mining to advanced symbol and feature recognition (Liu 2012), from a pure sentiment analysis to a sentiment and subjective analysis (Pang \& Lee 2004), from machine learning or lexicon-based approaches to more advanced hybrid methods (SerranoGuerrero et al. 2015) and from sentiment orientation with only two directions (e.g. positive and negative) coarse measurement scale to a fine grained classification (Fink et al. 2011). However, limited sentiment analysis research has been conducted from a spatial and temporal perspective. This study tested a research question of whether sentiment polarity on Twitter exhibits specific time-location patterns.

The aim of this paper is to investigate the spatial and temporal distribution of georeferenced Twitter sentiment polarity within Bentley campus, Curtin University in Perth Western Australia. The campus was divided into six zones - science and engineering buildings, social science buildings, library, lecture theatre, dormitory, entertainment and parking areas and four periods of time - beginning of the semester, middle of the semester, end of semester and after examination to investigate how Twitter sentiments vary across different zones and time periods. The Starlight Visual Information System ${ }^{1}$ was used to conduct a sentiment analysis. The Feature Manipulation Engine $\left(\mathrm{FME}^{2}\right)$ was employed to convert non-spatial files into spatial and temporal feature class.

\footnotetext{
1 http://starlight.pnnl.gov.

2 http://www.safe.com.
} 
The sentiment polarity patterns across six spatial zones and four temporal zones were mapped using the $\mathrm{ArcGIS}^{3}$ software.

The paper is structured as follows. Section 2 presents the research context including a review of the relevant research literature associated with methods for a Twitter sentiment analysis. The research method in Section 3 describes the overall approach taken in this research. Section 4 presents the results, and the paper concludes with a discussion of key findings and implications of our observations on different Twitter topics in Section 5.

\section{Related work}

Sentiment analysis is the Natural Language Processing work, which involves opinion detection and classification of attitudes in texts (Balahur et al. 2014). Numerous studies have been conducted for automatically detecting opinions and emotions. This section summarised these studies into two categories.

\section{Sentiment classification trends}

The early studies of sentiment classification are mostly based on text mining techniques. Opinion was classified into positive/negative or positive/negative/ neutral. It can be simple two, five or even eleven point scale depending on the complexity of a task (Taboada et al. 2011; Pang \& Lee 2008; Pang \& Lee 2004; Whitelaw et al. 2005). Human language tends to be subjective. The same sentence in different tones or contexts could in different emotional states. It creates a great challenge to identify the affective state or intended emotional communication (Sarvabhotla et al. 2011; Pang \& Lee 2004; Wilson et al. 2005). Therefore, subjectivity analysis can go beyond simple category of positive, negative or neutral (Liu 2012). Some studies focus on detecting ironic and sarcastic content of texts. However, there is a huge debate of how to formally define irony and sarcasm, which add another dimension of subjectivity analysis (Reyes \& Rosso 2012). Except extracting polarity of a given text, more fine-grained methods have been developed to detect emotion or opinions from symbols, such as emoticons (e.g. '(-), ' $\odot$ ') (Read 2005; Go et al. 2009) and visual features or images (Liu 2012). This progression of the studies has taken the sentiment analysis research to a new level.

\section{Classification methods}

In order to perform different sentiment classification tasks, various sentiment algorithms were developed (Medhat et al. 2014; Serrano-Guerrero et al. 2015).

\footnotetext{
${ }^{3}$ http://www.esri.com/software/arcgis.
} 
Medhat et al. (2014) grouped the SA into two categories: machine learning and lexicon-based approaches (see Figure 1). Generally, machine-learning methods were used to automatically discover sentiment polarity pattern rules in large data in order to learn opinions or emotions of given texts or features. A variety of algorithms have been developed (Ye et al. 2009; Rushdi Saleh et al. 2011). Most algorithms fall into the category of supervised machine learning. For example, Rushdi Saleh et al. (2011) applied Support Vector Machines (SVM) to detect whether the opinion expressed in a document is positive or negative about a given topic using several weighting schemes. Ye et al. (2009) compared Naïve Bayes, SVM and the character based N-gram model for analysing the sentiment of travel blogs for seven popular travel destinations in US and Europe. The SVM and $\mathrm{N}$-gram approach was found to outperform the Naïve Bayes approach with accuracies reaching to at least $80 \%$. Balahur (2013) developed an unsupervised method especially for a Twitter data sentiment analysis using the SVM. The major contribution of the study is to employ methods in normalising Tweet language, including higher order n-grams to spot modifications in sentiment polarity articulated and selecting features using simple heuristics.

Lexicon-based approaches focus on measuring subjectivity and opinions in texts using Semantic orientation (SO) (Osgood et al. 1957), which capture orientations of opinions (positive or negative) and strengths or degrees of orientation (Taboada et al. 2011). Sentiment lexicons are the key for this type

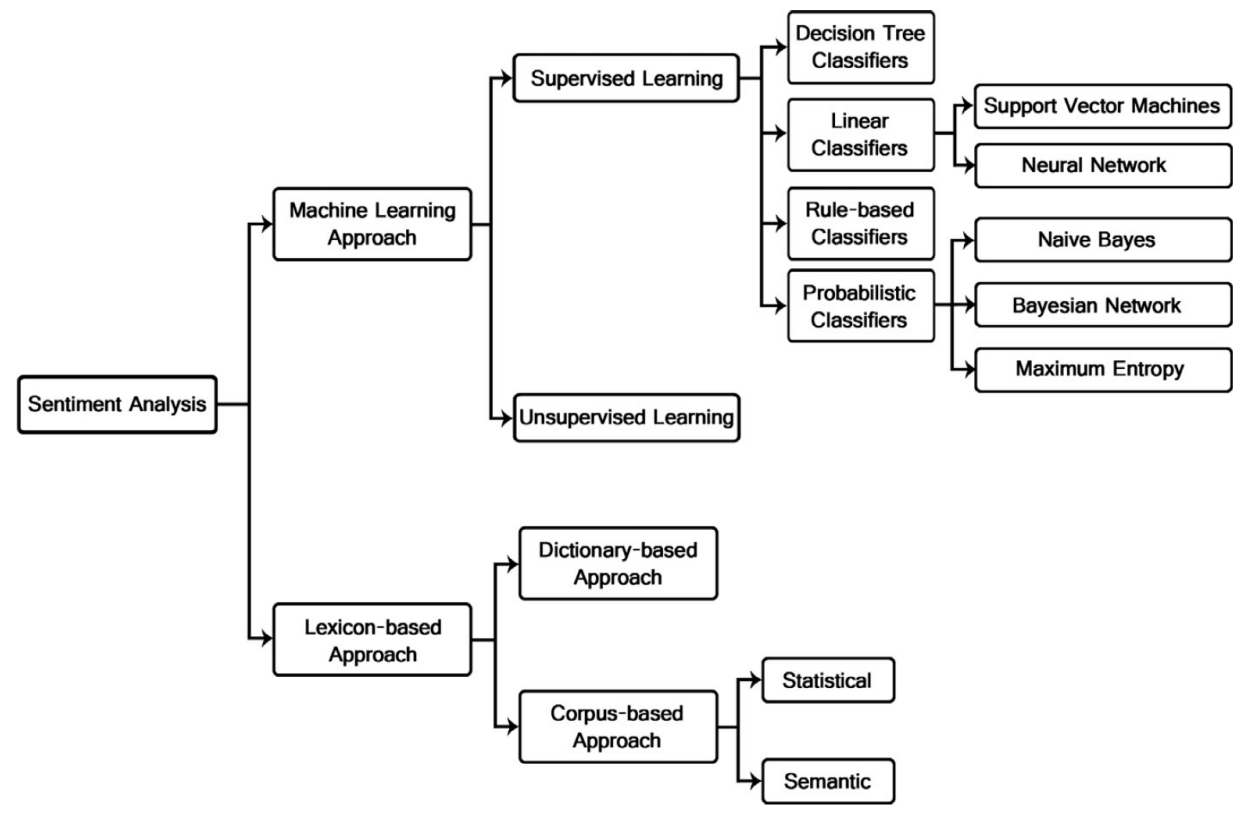

Figure 1: Sentiment classification techniques (Source: Medhat et al. 2014). 
of methods. For example, Paltoglou and Thelwall (2012) proposed a lexiconbased approach to identify whether a text conveys negative or positive attitudes and to estimate the level of emotional intensity of a text in social media and microblogging environments. They added extensive linguistically functionalities (negation/capitalization detection, intensifier/diminisher detection and emoticon/exclamation detection) to the traditional classifiers such as Support Vector Machines (SVM), Maximum Entropy classifier and Naive Bayes classifiers. Khan et al. (2014) utilised a hybrid system framework, which contains unsupervised learning algorithms and a dictionary-based method named Twitter Opinion Mining Framework (TOM). This method applied a variety of techniques for Twitter analysis and classification including a hybrid scheme of Enhanced Emoticon Classifier (EEC), SentiWordNet Classifier (SWNC) and an improved polarity classifier (IPC) using a list of positive/negative words. The findings reveal that the proposed algorithm resolved previous technical issues and increased the classification accuracy, effectively reduced the number of classified neutrals. Dacres et al. (2013) conducted a topic analysis and sentimental analysis in understanding the contents of Tweets and trends posted over a 10-day period using machine learning and natural language processing techniques. The researchers examined and compared the commenly-used Data Science Toolkit's text2sentiment4, which is based on different methods, such as sentiment lexicon (Nielsen 2011), the lexicon-based but data-driven hybrid SentiStrength (Thelwall et al. 2012), and Charrerbox's Sentimental API (Purver \& Battersby 2012). In Dacres et al. (2013) analysis, best result was achieved by the machine learning method (Charrerbox's Sentimental API) with $84 \%$ accuracy. In our study, we have also adopted a machine learning method using Subspace Transformation (TRUST) engine in Starlight for vector space modelling and supervised learning for a sentiment analysis.

\section{Methods}

\section{Study area and data collection methods}

\section{Study Area}

The developed content analysis methods were implemented using Tweets within the area of $1 \mathrm{~km}$ buffer around Curtin Bentley campus boundary (see Figure 2). Curtin University is one of the largest universities in Australia. It has more 60,000 students enrolled each year (OFFICE OF STRATEGY AND PLANNING 2014). The Bentley campus, as the main campus of Curtin University, is located about six $\mathrm{km}$ southeast from the Perth CBD. It covers 116 hectares with a variety of facilities, such as a library, lecture theatres, teaching rooms, cafés, dormitories and parking areas (Curtin University 2015). 


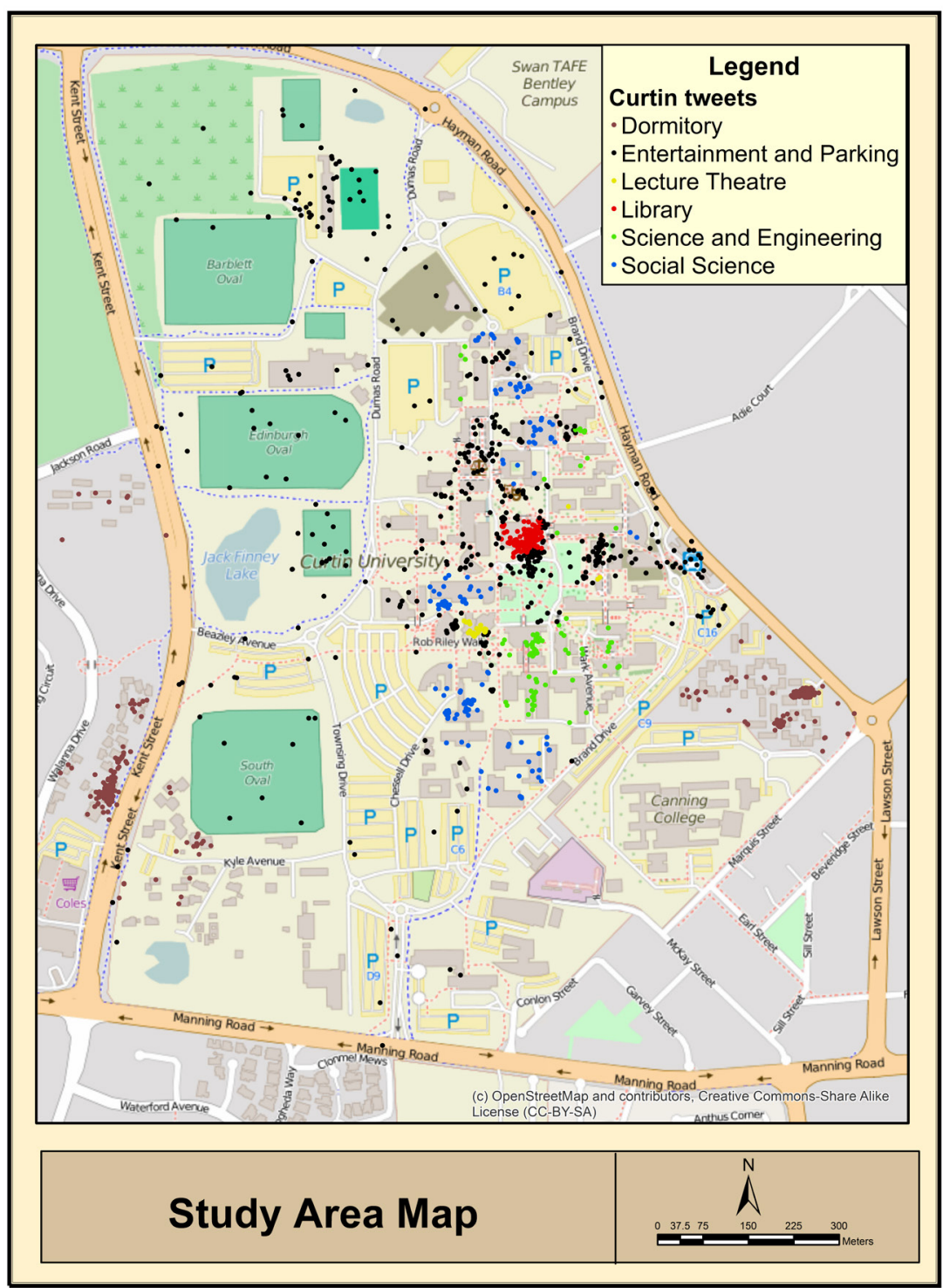

Figure 2: Curtin University Area Map (Map data (c) OpenStreetMap contributors).

\section{Data Gathering And Pre-processing}

Because the objective of this paper is to understand spatial and temporal patterns of georeferenced Tweets' emotional polarity, we only used geotagged Tweets posted between 12 May 2014 and 5 Jan 2015 within the area of $1 \mathrm{~km}$ buffer around Curtin Bentley campus boundary as JSON files via Twitter API 
and converted to XML format using the Ruby built-in JSON library. More than 5,000 Tweets were downloaded from Twitter API during the study period but around one third of them were located outside the Curtin University boundary. After removing the ones outside Curtin University boundary, there were a total of 3,172 Tweets gathered. These XML files of Tweets consist of both geo-location information and attribute information such as text of Tweets, time created at and geolocation, which are directly relevant to our research. Tweets of non-English languages were removed for this study in order to simplify the analysis, leaving 3,097 Tweets in the dataset. In our study, location associated with each Tweet is in the format of $(\mathrm{x}, \mathrm{y})$ coordinates, which were automatically captured using built-in Global Positioning System (GPS) receivers in mobile or tablet applications, such as a smart phone. This is the exact location where a user Tweeted. The accuracy of location recorded by GPS is usually around a few meters. However, if the location captured by triangulation outside a cellular network, the accuracy can range between $30-3000 \mathrm{~m}$, subjecting to the cell distribution ( $\mathrm{Li}$ et al., 2013). These geotagged Twitter data were imported into a geodatabase using GIS software, such as, FME and ArcGIS, as point features, Curtin Buildings and boundary geography files were extracted from Open Street Map ${ }^{4}$ and converted into the same geodatabase using the ArcGIS software.

\section{Sentiment analysis methods}

There are three levels of sentimental analysis -1 ) document level sentiment classification; 2) sentence level sentiment classification; and 3) aspect level sentiment analysis (Liu 2012). This research chose the sentence level sentiment classification. The sentence level sentiment classification is suitable because of its assumption that each sentence contains only one entity or one aspect of entity in many cases (Liu 2012). Each Tweet has a limitation of 140 characters in order to ensure that information posted on Twitter is straight forward to the theme. As a result, it is more suitable to perform a sentence level sentiment analysis on Tweets. This study carried out a sentiment analysis by using the Starlight's Sentiment Analyzer function in Starlight Data Engineer (SDE), which adopted the Boeing Text Representation using Subspace Transformation (TRUST) engine for vector space modelling and text summarisation (Simoff et al. 2008). The input for Sentiment Analyzer is XML files. Sentiment Analyzer analyses individual words of a Tweet and calculates a score of sentiment orientation. It returns statistics from the sentiment analysis, such as, sentimentTotal, sentimentDiff, sentimentScore, wordCount, sentimentNegative, sentimentPositive. In this study, sentimentDiff is the sentiment orientation of Tweets. SentimentDiff is the result of sentimentPositive subtracting sentimentNegative (Liu 2012). If sentimentDiff is positive number, it means this text hold

\footnotetext{
${ }^{4}$ http://www.openstreetmap.org
} 
a positive attitude. If sentimentDiff is negative, it means the text expresses a negative opinion. These simple statistics returned from the Sentiment Analyzer are defined as the followings:

- SentimentTotal: the sum of sentimentPositive and sentimentNegative.

- SentimentDiff: sentimentPositive subtract sentimentNegative.

- SentimentScore:sentimentDiff divided by WordCount.

- WordCount: the total number of words in the text.

- SentimentNegative: each negative term in the text represent by 1 . SentimentNegative is the total number of negative words in the text. For example, text 'such a bad day! Everything is wrong.' 'Bad' and 'wrong' makes the sentimentNegative to be 2 .

- SentimentPositive: each positive term in the text represents by 1 . SentimentPositive is the total number of positive words in the text. For instance, sentence 'study hard and the review is quite efficient', 'hard' and 'efficient' makes the sentimentPositive score to be 2 .

From the analysis above, the range of sentiment orientation scores were derived from this study from 5 to -6 . The sentiment category is shown in Table 1 . The scale we used does not consider severity of individual words, but their frequency.

Sentiment orientation scores ranging from 4 to 5 represent Tweets expressing very positive sentiment and sentiment orientation scores ranging from 1 to 3 mean Tweets holding positive sentiments. Sentiment orientation score 0 means Tweets do not express any opinions. Sentiment orientation scores from -4 to -6 represent Tweets holding very negative sentiment while sentiment orientation scores ranging from -1 to -3 mean negative attitudes.

\section{Spatial and temporal comparison of Twitter sentiment polarity patterns}

We divided Curtin Bentley University campus into six spatial zones and four time periods (see Table 2). The spatial distribution of georeferenced Tweets

\begin{tabular}{|l|c|}
\hline \multicolumn{1}{|c|}{ Sentiment Category } & Sentiment orientation scores \\
\hline Very Positive & $4-5$ \\
\hline Positive & $1-3$ \\
\hline Natural & 0 \\
\hline Negative & $(-1)-(-3)$ \\
\hline Very Negative & $(-4)-(-6)$ \\
\hline
\end{tabular}

Table 1: Sentiment category. 


\begin{tabular}{|l|l|}
\hline \multicolumn{1}{|c|}{ Study Period Category } & \multicolumn{1}{c|}{ Time Span } \\
\hline Beginning of the semester & 28 July 2014 - 7 September 2014 \\
\hline Middle of the semester & $\begin{array}{l}\text { 6 May 2014 - 18 May 2014 and 8 September 2014 - } \\
\text { 19 October 2014 }\end{array}$ \\
\hline End of the semester & $\begin{array}{l}\text { 19 May 2014 - 27 June 2014 and 20 October 2014 - } \\
\text { 28 November 2014 }\end{array}$ \\
\hline After examination & $\begin{array}{l}\text { 28 June 2014 - 27 July 2014 and 29 November 2014 - } \\
\text { 5 January 2015 }\end{array}$ \\
\hline
\end{tabular}

Table 2: Study period category.

among six zones was derived using ArcGIS software. In addition, the ArcGIS software was used to assign all Tweets with time span information and exported to an excel spread sheet for further analyses.

\section{Research Hypothesis}

\section{- Spatial difference}

Tweets posted in different campus locations are various in sentiments. For example, Tweets posted in Entertainment and parking area may be more positive while Tweets posted in study areas may be more negative.

- Temporal difference

Adnan et al. (2012) conducted research on student stress levels at the beginning and the end of the semester and found the stress level of university students varies throughout a semester. Generally speaking, students felt more stressful at the end of the semester than at the beginning of the semester. Based on this study, we proposed a hypothesis that more positive Tweets are posted at the beginning of semester and after examination compared with the ones posted at the middle of the semester and at the end of the semester.

\section{Results}

\section{Overall distribution of the Tweets by sentiments}

Three thousand and ninety-seven Tweets were loaded into the Starlight Data Engineer for a sentiment analysis and output Tweets were assigned sentiment polarity. Then output Tweets from Starlight were further processed in FME to be converted into feature classes. Table 3 illustrates the overall distribution of the Tweets by sentiments. Around $45 \%$ of Tweets contain neutral opinions, such as 'I'm at Curtin University'. Besides, the number of Tweets holding positive 


\begin{tabular}{|l|c|r|}
\hline \multicolumn{1}{|c|}{ Sentiments } & Score range & Number of Tweets \\
\hline Very positive & $4-6$ & 32 \\
\hline Positive & $1-3$ & 1,091 \\
\hline Neutral & 0 & 1,383 \\
\hline Negative & $(-1)-(-3)$ & 577 \\
\hline Very Negative & $(-4)-(-6)$ & 14 \\
\hline
\end{tabular}

Table 3: Overall distributions.

opinions is nearly as twice as Tweets of negative opinions. Fourteen Tweets contain very negative feelings while thirty-two Tweets have very positive opinions.

\section{Spatial distribution of sentiment polarity patterns}

As it is showed in Figure 3 and Table 4, most Tweets were posted in the entertainment and parking and dormitory area. Tweets posted in the science and engineering area do not have very negative opinions, while Tweets posted in lecture theatre and library do not contain very positive opinions. Tweets posted in social science areas have the highest percentage of positive Tweets (42.1\%), while Tweets gathered in the library area have the largest percentage of negative opinions (21.8\% of total Tweets in the area). About $20.7 \%$ of total Tweets posted in science and engineering area hold negative opinions, while $18 \%$ of Tweets in social science are negative. Therefore the descriptive data potentially indicates that students or staff in the science and engineering areas could feel slightly more negative compared with students or staff in the social science areas.

\section{Temporal distribution of sentiment polarity patterns}

Sentiment orientation at different study time zones is showed in Table 5. It is interesting to note that the largest percentage of negative feeling actually occur at the beginning of semester, which is $21.6 \%$. This is different from our hypothesis. The percentage of negative sentiments decreases from the beginning of the semester zone to after exam zone, reaching to smallest number of negative opinions (0.08) after examination. The percentage of very negative Tweets is roughly the same throughout all four study zones. After examination time zone holds the largest percentage of positive Tweets, which is align with the research hypothesis.

The library area contains a large cluster of Tweets (See Figure 4), which shows an interesting temporal pattern. At the beginning of the semester, still more positive Tweets occurred than negative ones in the library area. However, as a semester goes, more negative Tweets were posted in the library area. The percentage of positive Tweets in each temporal zone decreased over time gradually. We also summarised the number of negative opinions on Twitter across five 


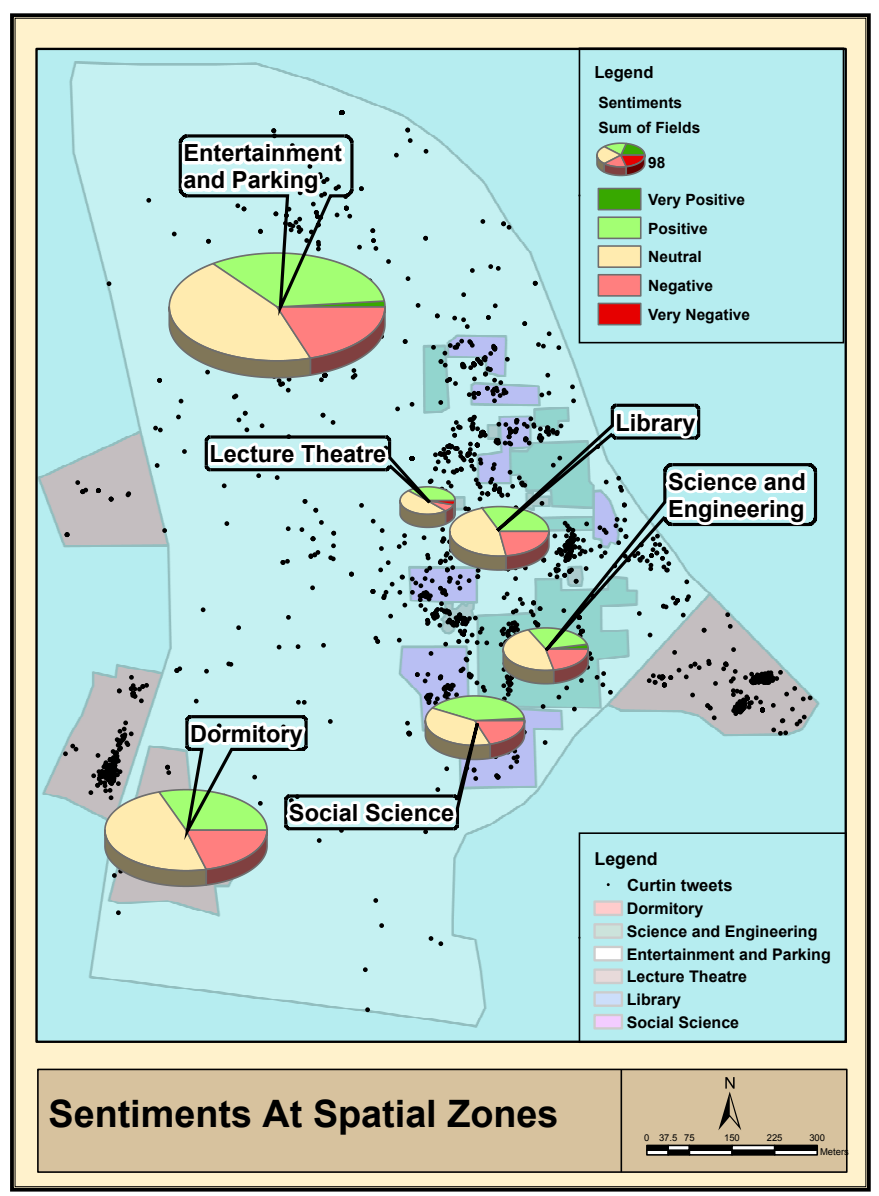

Figure 3: Sentiments at spatial zones.

\begin{tabular}{|l|c|c|c|c|c|r|}
\hline Spatial zones & $\begin{array}{c}\text { Very } \\
\text { Positive }\end{array}$ & Positive & Neutral & Negative & $\begin{array}{c}\text { Very } \\
\text { Negative }\end{array}$ & Total \\
\hline $\begin{array}{l}\text { Science and } \\
\text { Engineering }\end{array}$ & $5(2.7 \%)$ & $59(31.4 \%)$ & $85(45.2 \%)$ & $39(20.7 \%)$ & $0(0 \%)$ & 188 \\
\hline Social Science & $3(1.1 \%)$ & $110(42.1 \%)$ & $99(37.9)$ & $47(18 \%)$ & $2(0.8 \%)$ & 261 \\
\hline Library & $0(0 \%)$ & $85(32.6 \%)$ & $118(45.2 \%)$ & $57(21.8 \%)$ & $1(0.4 \%)$ & 261 \\
\hline Lecture Theatre & $0(0 \%)$ & $22(40.0 \%)$ & $27(49.1 \%)$ & $4(7.3 \%)$ & $2(3.6 \%)$ & 55 \\
\hline Dormitory & $5(1.6 \%)$ & $260(32.1 \%)$ & $387(47.8 \%)$ & $156(19.3 \%)$ & $2(0.2 \%)$ & 810 \\
\hline $\begin{array}{l}\text { Entertainment } \\
\text { and Parking }\end{array}$ & $19(1.2 \%)$ & $555(36.4 \%)$ & $667(43.8 \%)$ & $275(18.1 \%)$ & $7(0.5 \%)$ & 1523 \\
\hline
\end{tabular}

Table 4: Sentiments for spatial zones.

*The percentage of very positive Tweets in the Science and Engineering area. 


\begin{tabular}{|l|c|c|c|c|c|c|}
\hline \multicolumn{1}{|c|}{ TimeZones } & $\begin{array}{c}\text { Very } \\
\text { Positive }\end{array}$ & Positive & Neutral & Negative & $\begin{array}{c}\text { Very } \\
\text { Negative }\end{array}$ & Total \\
\hline $\begin{array}{l}\text { Beginning of } \\
\text { semester }\end{array}$ & $12(1.5 \%)$ & $275(35.6 \%)$ & $316(40.9 \%)$ & $167(21.6 \%)$ & $3(0.4 \%)$ & 773 \\
\hline $\begin{array}{l}\text { Middle of } \\
\text { semester }\end{array}$ & $11(0.9 \%)$ & $407(33.6 \%)$ & $542(44.8 \%)$ & $244(20.2 \%)$ & $6(0.5 \%)$ & 1210 \\
\hline End of semester & $7(0.8 \%)$ & $334(36.0 \%)$ & $431(46.5 \%)$ & $151(16.3 \%)$ & $4(0.4 \%)$ & 927 \\
\hline After exam & $2(1.1 \%)$ & $76(40.4 \%)$ & $94(50.0 \%)$ & $15(8.0 \%)$ & $1(0.5 \%)$ & 188 \\
\hline
\end{tabular}

Table 5: Sentiment polarity of temporal zones.

\begin{tabular}{|l|c|c|r|r|r|r|}
\hline \multicolumn{1}{|c|}{ Time Zones } & $\begin{array}{c}\text { Very } \\
\text { Positive }\end{array}$ & Positive & Neutral & Negative & $\begin{array}{c}\text { Very } \\
\text { Negative }\end{array}$ & Total \\
\hline $\begin{array}{l}\text { Beginning of the } \\
\text { semester }\end{array}$ & 0 & $12\left(35 \%^{*}\right)$ & $15(44 \%)$ & $7(21 \%)$ & 0 & 34 \\
\hline $\begin{array}{l}\text { Middle of the } \\
\text { semester }\end{array}$ & 0 & $34(33 \%)$ & $47(46 \%)$ & $22(21 \%)$ & 0 & 103 \\
\hline End of the semester & 0 & $38(31 \%)$ & $56(46 \%)$ & $28(23 \%)$ & $1(1 \%)$ & 123 \\
\hline After examination & 0 & $1(100 \%)$ & 0 & 0 & 0 & 1 \\
\hline
\end{tabular}

Table 6: Sentiment polarity of temporal zones in the library area. *The percentage of positive Tweets in the beginning of semester

\begin{tabular}{|l|r|r|r|r|r|r|r|}
\hline \multicolumn{1}{|c|}{$\begin{array}{c}\text { Time } \\
\text { Zones }\end{array}$} & Dormitory & $\begin{array}{c}\text { Science and } \\
\text { Engineering }\end{array}$ & $\begin{array}{c}\text { Entertainment } \\
\text { and Parking }\end{array}$ & $\begin{array}{r}\text { Lecture } \\
\text { Theatre }\end{array}$ & Library & $\begin{array}{c}\text { Social } \\
\text { Science }\end{array}$ & Total \\
\hline $\begin{array}{l}\text { Beginning } \\
\text { the of } \\
\text { semester }\end{array}$ & 49 & 11 & 86 & 1 & 7 & 13 & 167 \\
\hline $\begin{array}{l}\text { Middle } \\
\text { of the } \\
\text { semester }\end{array}$ & 72 & 13 & 116 & 3 & 22 & 19 & 245 \\
\hline $\begin{array}{l}\text { End of the } \\
\text { semester }\end{array}$ & 31 & 14 & 63 & 0 & 28 & 15 & 151 \\
\hline $\begin{array}{l}\text { After } \\
\text { examination }\end{array}$ & 4 & 1 & 10 & 0 & 0 & 0 & 15 \\
\hline
\end{tabular}

Table 7: The number of negative opinions on Twitter over space and time.

spatial zones and four temporal zones (see Table 7). Interestingly, except the science and engineering area, the number of negative opinions dropped at the end of semester in the other three areas. Certain temporal patterns of Tweeter polarity only occurred at specific locations. This may indicate that Twitter sentiments are time-location specific and they might depend on the activities conducted at certain locations. 


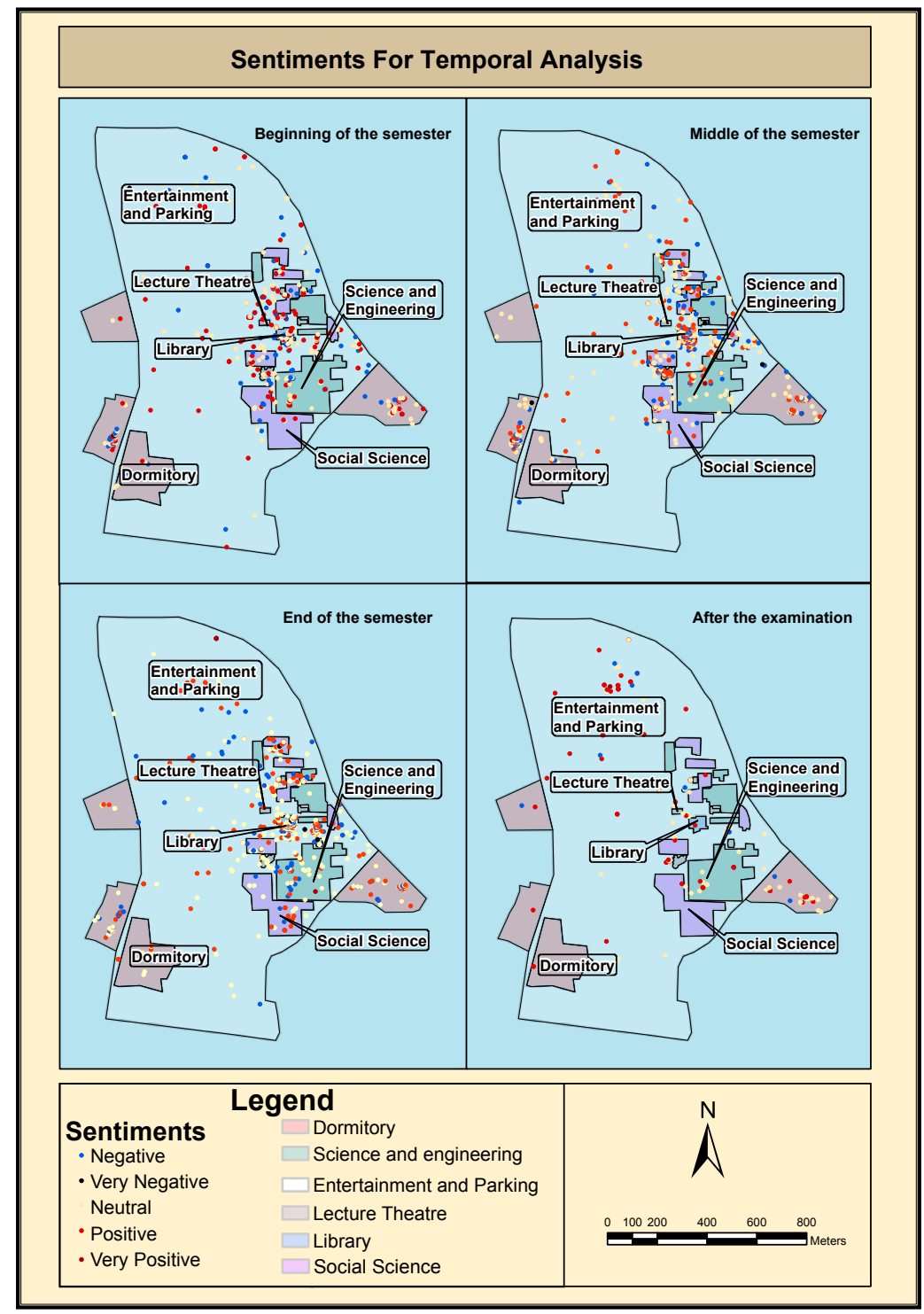

Figure 4: The distribution of sentiment polarity over time.

\section{Concluding remarks}

This study presents methods for analysing spatial and temporal patterns of Twitter sentiment polarity at Curtin University. By using this case study, we hope the results can provide some insights into understanding the spatial and 
temporal variation of students and staff's sentiment polarity. We separated Tweets into five different spatial zones and four time zones and tested a research question of whether sentiment polarity on Twitter exhibits specific time-location patterns.

Interestingly, although the number of positive Tweets posted in the entertainment and parking area were larger than the ones posted in the study areas, the highest percentage of positive Tweets were found in social science with over $40 \%$. Besides, science and engineering and dormitory areas had the highest percentage of negative postings (both over 19\%). It indicates that the sample of students in social science students tended to post twitter messages containing a higher ratio of positive-to-negative words than the sample of students in science and engineering in Curtin university. In addition, a trend of increasing number of negative Tweets was identified in library and science and engineering areas as a semester goes from the beginning to the end, reaching a peak around the exam period. While for the entertainment, sport and dormitory area, the percentage of negative Tweets dropped at the end of semester. This could mean that negative feelings might be associated with exam induced stress and study workload (Adnan et al. 2012).

The spatial and temporal sentiment analysis used geotagged Twitter data, which allow a sentiment polarity analysis at a fine-grained level. This method can be applied in many areas, such as polls (Wang et al. 2012a), consumer opinions concerning brands (Jansen et al. 2009), stock market performance (Bollen et al. 2011), crime prediction (Wang et al. 2012b) and tourism information (Shimada et al. 2011). However, there are a few limitations to be considered when making conclusions from this study. For example, outputs from the sentiment analysis in Starlight Data engineer are not perfectly accurate. For example, emotion tokens cannot be analysed in the sentiment analysis. Some emotion tokens, such as ' $\odot$ ' and ' $\odot$ ', actually express very obvious attitudes. But our methods in the sentiment analysis cannot process them. Besides, our sentiment analysis methods cannot handle sarcasm properly. For example, a Tweet of 'Well done. You have forgotten your umbrella' expresses a negative feeling, but the sentiment analysis misclassified it as positive. Some Tweets do not have sentiment words but they imply some emotions, which the sentiment analysis could misclassify them. For instance, 'Sleeping pattern is sooo screwed up, no more 4am' appears to be a negative sentiment, but the sentiment analysis classified it as neutral. In the future, this study will adopt more advanced algorithms, such as methods developed by Liu (2012), Katz et al. (2015) and Poria et al. (2014) for the sentiment analysis. In addition, in this study, we tested our hypotheses in only one university. We will collect Twitter data of major universities in Western Australia and conduct a comparison study in the future. In addition, we will develop a better measure of sentiment polarity, which will take both severity and frequency of individual words into account in the future. 


\section{Acknowledgements}

The authors are very grateful to Curtin University who provides a research grant to support this study. Constructive comments from the editor and anonymous reviewers have significantly improved this paper. We would also like to thank Dr Wei Liu to share Twitter data with the research team, The views expressed in this article are those of the authors, and do not necessarily reflect the policies of any organisation.

\section{References}

Adnan, N., Murat, Z. H., Abdul Kadir, R. S. S., \& Noradibah Hj Mohamad, Y. 2012. University students stress level and brainwave balancing index: Comparison between early and end of study semester. Research and Development (SCOReD), 2012 IEEE Student Conference, 5-6 Dec., 2012. Pulau Pinang, pp. 42-47.

Balahur, A. 2013. Sentiment analysis in social media texts. Citeseer.

Balahur, A., Mihalcea, R., \& Montoyo, A. 2014. Computational approaches to subjectivity and sentiment analysis: Present and envisaged methods and applications. Computer Speech \& Language, 28: 1-6.

Bollen, J., Mao, H., \& Zeng, X. 2011. Twitter mood predicts the stock market. Journal of Computational Science, 2: 1-8.

Curtin University. 2015. About [Online]. Available at: http://about.curtin.edu. au/campus-locations/directions/ [Accessed March 26 2015].

Dacres, S., Haddadi, H., \& Purver, M. 2013. Topic and Sentiment Analysis on OSNs: a Case Study of Advertising Strategies on Twitter.

Fink, C. R., Chou, D. S., Kopecky, J. J., \& Llorens, A. J. 2011. Coarse- and FineGrained Sentiment Analysis of Social Media Text. Johns Hopkins APL Technical Digest, 30: 22-30.

Go, A., Bhayani, R., \& Huang, L. 2009. Twitter sentiment classification using distant supervision. CS224N Project Report, Stanford: 1-12.

Jansen, B. J., Zhang, M., Sobel, K., \& Chowdury, A. 2009. Twitter power: Tweets as electronic word of mouth. Journal of the American Society for Information Science and Technology, 60: 2169-2188.

Katz, G., Ofek, N., \& Shapira, B. 2015. ConSent: Context-based sentiment analysis. Knowledge-Based Systems.

Khan, F. H., Bashir, S., \& Qamar, U. 2014. TOM: Twitter opinion mining framework using hybrid classification scheme. Decision Support Systems, 57: 245-257.

Li, L., Goodchild, M. F., \& Xu, B. 2013. Spatial, temporal, and socioeconomic patterns in the use of Twitter and Flickr. cartography and geographic information science, 40: 61-77. 
Liu, B. 2012. Sentiment Analysis and Opinion Mining. San Rafael: Morgan \& Claypool Publishers.

Medhat, W., Hassan, A., \& Korashy, H. 2014. Sentiment analysis algorithms and applications: A survey. Ain Shams Engineering Journal, 5: 1093-1113.

Nielsen, F. A. 2011. A new ANEW: Evaluation of a word list for sentiment analysis in microblogs.

Office of Strategy and Planning. 2014. Curtin University Student Statistics 2009-2013 [Online]. Available at: https://planning.curtin.edu.au/stats/students2009-2013.cfm [Accessed March 26 2015].

Osgood, C. E., Suci, G., \& Tannenbaum, P. 1957. The measurement of meaning, 81.

Paltoglou, G., \& Thelwall, M. 2012. Twitter, MySpace, Digg: Unsupervised sentiment analysis in social media. ACM Transactions on Intelligent Systems and Technology, 3.

Pang, B., \& Lee, L. 2004. A sentimental education: Sentiment analysis using subjectivity summarization based on minimum cuts. In: Proceedings of the 42nd annual meeting on Association for Computational Linguistics, 2004. Association for Computational Linguistics, p. 271.

Pang, B., \& Lee, L. 2008. Opinion mining and sentiment analysis. Foundations and trends in information retrieval, 2: 1-135.

Poria, S., Gelbukh, A., Cambria, E., Hussain, A., \& Huang, G.-B. 2014. EmoSenticSpace: A novel framework for affective common-sense reasoning. Knowledge-Based Systems, 69: 108-123.

Read, J. 2005. Using emoticons to reduce dependency in machine learning techniques for sentiment classification. In: Proceedings of the ACL Student Research Workshop, 2005. Association for Computational Linguistics, pp. 43-48.

Reyes, A., \& Rosso, P. 2012. Making objective decisions from subjective data: Detecting irony in customer reviews. Decision Support Systems, 53: 754-760.

Rushdi Saleh, M., Martín-Valdivia, M. T., Montejo-Ráez, A., \& Ureña-López, L. A. 2011. Experiments with SVM to classify opinions in different domains. Expert Systems with Applications, 38: 14799-14804.

Sarvabhotla, K., Pingali, P., \& Varma, V. 2011. Sentiment classification: a lexical similarity based approach for extracting subjectivity in documents. Information Retrieval, 14: 337-353.

Serrano-Guerrero, J., Olivas, J. A., Romero, F. P., \& Herrera-Viedma, E. 2015. Sentiment analysis: A review and comparative analysis of web services. Information Sciences, 311: 18-38.

Shimada, K., Inoue, S., Maeda, H., \& Endo, T. 2011. Analyzing tourism information on twitter for a local city. In: Software and Network Engineering (SSNE), 2011. First ACIS International Symposium on, 2011. IEEE, pp. 61-66.

Simoff, S., Böhlen, M. H., \& Mazeika, A. 2008. Visual data mining: theory, techniques and tools for visual analytics, Springer Science \& Business Media.

Taboada, M., Brooke, J., Tofiloski, M., Voll, K., \& Stede, M. 2011. Lexicon-based methods for sentiment analysis. Computational linguistics, 37: 267-307. 
Thelwall, M., Buckley, K., \& Paltoglou, G. (2012). Sentiment strength detection for the social web. Journal of the American Society for Information Science and Technology, 63: 163-173.

Wang, H., Can, D., Kazemzadeh, A., Bar, F., \& Narayanan, S. 2012a. A system for real-time twitter sentiment analysis of 2012 us presidential election cycle. In: Proceedings of the ACL 2012 System Demonstrations. Association for Computational Linguistics, pp. 115-120.

Wang, X., Gerber, M. S., \& Brown, D. E. 2012b. Automatic crime prediction using events extracted from twitter posts. In: Social Computing, BehavioralCultural Modeling and Prediction. Springer.

Whitelaw, C., Garg, N., \& Argamon, S. 2005. Using appraisal groups for sentiment analysis. In: Proceedings of the 14th ACM international conference on Information and knowledge management, 2005. ACM, pp. 625-631.

Wilson, T., Wiebe, J., \& Hoffmann, P. 2005. Recognizing contextual polarity in phrase-level sentiment analysis. In: Proceedings of the conference on human language technology and empirical methods in natural language processing, 2005. Association for Computational Linguistics, pp. 347-354.

Ye, Q., Zhang, Z., \& Law, R. 2009. Sentiment classification of online reviews to travel destinations by supervised machine learning approaches. Expert Systems with Applications, 36: 6527-6535. 